Open Access

\title{
Microdeletions in 9q33.3-q34.11 in five patients with intellectual disability, microcephaly, and seizures of incomplete penetrance: is STXBP1 not the only causative gene?
}

Julia K. Ehret ${ }^{1 \dagger}$, Hartmut Engels ${ }^{1 *+}$, Kirsten Cremer ${ }^{1}$, Jessica Becker ${ }^{1,2}$, Johannes P. Zimmermann ${ }^{1}$, Eva Wohlleber ${ }^{1,3}$, Ute Grasshoff4, Eva Rossier ${ }^{4,5}$, Michael Bonin ${ }^{4,6}$, Elisabeth Mangold ${ }^{1}$, Andrea Bevot ${ }^{7}$, Stefanie Schön ${ }^{1,8}$,

Stefanie Heilmann-Heimbach ${ }^{1,2}$, Nicola Dennert ${ }^{1}$, Michèle Mathieu-Dramard ${ }^{9}$, Elodie Lacaze ${ }^{10}$, Ghislaine Plessis ${ }^{10}$, Alain de Broca ${ }^{11}$, Guillaume Jedraszak ${ }^{9}$, Benno Röthlisberger ${ }^{12}$, Peter Miny ${ }^{13}$, Isabel Filges ${ }^{13}$, Andreas Dufke ${ }^{4}$, Joris Andrieux ${ }^{14 \dagger}$, Jennifer A. Lee $e^{1,2,15 \dagger}$ and Alexander M. Zink ${ }^{1 \dagger}$

\begin{abstract}
Background: Most microdeletions involving chromosome sub-bands 9q33.3-9q34.11 to this point have been detected by analyses focused on STXBP1, a gene known to cause early infantile epileptic encephalopathy 4 and other seizure phenotypes. Loss-of-function mutations of STXBP1 have also been identified in some patients with intellectual disability without epilepsy. Consequently, STXBP1 is widely assumed to be the gene causing both seizures and intellectual disability in patients with 9q33.3-q34.11 microdeletions.
\end{abstract}

Results: We report five patients with overlapping microdeletions of chromosome 9q33.3-q34.11, four of them previously unreported. Their common clinical features include intellectual disability, psychomotor developmental delay with delayed or absent speech, muscular hypotonia, and strabismus. Microcephaly and short stature are each present in four of the patients. Two of the patients had seizures. De novo deletions range from 1.23 to $4.13 \mathrm{Mb}$, whereas the smallest deletion of $432 \mathrm{~kb}$ in patient 3 was inherited from her mother who is reported to have mild intellectual disability. The smallest region of overlap (SRO) of these deletions in 9q33.3 does not encompass STXBP1, but includes two genes that have not been previously associated with disease, RALGPS1 and GARNL3.

Sequencing of the two SRO genes RALGPS1 and GARNL3 in at least 156 unrelated patients with mild to severe idiopathic intellectual disability detected no causative mutations. Gene expression analyses in our patients demonstrated significantly reduced expression levels of GARNL3, RALGPS1 and STXBP1 only in patients with deletions of the corresponding genes. Thus, reduced expression of STXBP1 was ruled out as a cause for seizures in our patient whose deletion did not encompass STXBP1.

Conclusions: We suggest that microdeletions of this region on chromosome $9 \mathrm{q}$ cause a clinical spectrum including intellectual disability, developmental delay especially concerning speech, microcephaly, short stature, mild dysmorphisms, strabismus, and seizures of incomplete penetrance, and may constitute a new contiguous gene deletion syndrome which cannot completely be explained by deletion of STXBP1.

Keywords: Microdeletion 9q33.3-q34.11, Intellectual disability, STXBP1, Contiguous gene syndrome, Haploinsufficiency

\footnotetext{
*Correspondence: hartmut.engels@uni-bonn.de

${ }^{\dagger}$ Equal contributors

'Institute of Human Genetics, University of Bonn, Sigmund-Freud-Strasse 25,

53105 Bonn, Germany

Full list of author information is available at the end of the article
}

\section{() Biomed Central}

(c) 2015 Ehret et al. Open Access This article is distributed under the terms of the Creative Commons Attribution 4.0 International License (http://creativecommons.org/licenses/by/4.0/), which permits unrestricted use, distribution, and reproduction in any medium, provided you give appropriate credit to the original author(s) and the source, provide a link to the Creative Commons license, and indicate if changes were made. The Creative Commons Public Domain Dedication waiver (http://creativecommons.org/publicdomain/zero/1.0/) applies to the data made available in this article, unless otherwise stated. 


\section{Background}

Previously, microdeletions affecting the chromosomal region 9q33.3-q34.11 have been reported in association with early infantile epileptic encephalopathy 4 (EIEE4, or Ohtahara syndrome, OMIM \#612164). The STXBP1 gene was identified as a causative gene and loss-offunction mutations of STXBP1 have been shown to cause EIEE4 [1], West syndrome [2], other seizure phenotypes [3], non-syndromic epilepsy [4], and intellectual disability (ID). De novo sequence mutations in STXBP1 have also been reported to be associated with ID without epilepsy [5].

Here we present the clinical and genetic characterization of five patients whose common clinical features include intellectual disability (ID), psychomotor developmental delay (DD) with delayed or absent speech, muscular hypotonia, strabismus, dysmorphisms and other recurrent findings. For these patients, we have identified overlapping microdeletions of chromosome 9q33.3-q34.11 by molecular karyotyping. The smallest region of overlap (SRO) concerning these common clinical features contains only two RefSeq genes, which has implications regarding the potential role STXBP1 may or may not play in our patients' phenotypes.

\section{Results}

\section{Case reports}

\section{Patient 1}

Patient 1 is the first and only child of healthy nonconsanguineous parents from Turkey with unremarkable family histories. She was born by vacuum extraction after 41 weeks of gestation with a birth weight of $3135 \mathrm{~g}$ $\left(10-25^{\text {th }}\right.$ centile, -1.1 S.D. $)$, a length of $49 \mathrm{~cm}\left(3^{\text {rd }}-10^{\text {th }}\right.$ centile, -1.7 S.D. $)$, and an OFC of $32 \mathrm{~cm}\left(<3^{\text {rd }}\right.$ centile, -2.6 S.D.). Postnatal adaptation was normal (APGAR 10/10, umbilical cord $\mathrm{pH}$ 7.26). Exotropia of the left eye and bilateral pes supinatus were noted at the age of 1 month. Between 6 and 10 months of age, she displayed clear motor delays and muscular hypotonia. Cranial ultrasound examination at 8 months gave normal results, except for asymmetry of lateral ventricles. Hearing was normal.

At the age of 10 months, her height and weight were normal $\left[69 \mathrm{~cm}\left(3 \mathrm{rd}-10^{\text {th }}\right.\right.$ centile, -1.6 S.D.); $10.5 \mathrm{~kg}$ $\left(90-97^{\text {th }}\right.$ centile, +1.6 S.D.)], but she remained microcephalic $\left[42.7 \mathrm{~cm}\left(<3^{\text {rd }}\right.\right.$ centile, -2.3 S.D. $\left.)\right]$. Clinical genetic examination at 11 months revealed some craniofacial dysmorphism with cranial asymmetry (flat right occiput), round face, telecanthus, upslanting palpebral fissures, epicanthic folds, short nose, thin upper lip, low-set ears with attached earlobes, and a short neck. Her fingers were tapered with ridges of both thumb and index finger nails (Fig. 1a, c). Pes equinus was noted. She showed muscular hypotonia, especially of the trunk and shoulder girdle. She was able to bring her hands together as well as to her mouth and feet, as well as grasp toys and transfer them to the opposite side of her body. However, she was unable to roll over or support her upper body in the prone position. At the age of 20 months she weighed $14 \mathrm{~kg}\left(97^{\text {th }}\right.$ centile, +2.0 S.D. $)$, her height was $78.5 \mathrm{~cm}\left(3^{\text {rd }}-10^{\text {th }}\right.$ centile, -1.8 S.D.), and her OFC was $46 \mathrm{~cm}\left(10-25^{\text {th }}\right.$ centile, -1.1 S.D.). In addition to the craniofacial dysmorphism described above she showed brachycephaly and a high frontal hairline. At the age of 20 months she was able to roll over but not to crawl. Language development was delayed with only few syllables. She showed strabismus and bruxism. Recurrent otitis was treated with transtympanic drains. The parents reported that EEG and ultrasonic examinations of the heart and kidneys were performed with normal results. Cerebral MRI scan at the age of 33 months showed a delay of myelination of about 3 months. At the age of 3 years, muscular hypotonia was very distinctive. The patient was unable to sit, stand, or walk. Her parents reported lack of pain sensation. She still displayed no seizures.

Conventional karyotyping and sequencing of the RPS6KA3 (RSK2) gene yielded normal results.

\section{Patient 2}

Patient 2 is the first child of healthy and unrelated parents. He was delivered spontaneously after gestational week $40+4$ with a birth weight of $2650 \mathrm{~g}\left(<3^{\text {rd }}\right.$ centile, -2.3 S.D.), a length of $47 \mathrm{~cm}\left(<3^{\text {rd }}\right.$ centile, -2.5 S.D. $)$, and an OFC of $31.5 \mathrm{~cm}\left(<3^{\text {rd }}\right.$ centile, -3.3 S.D. $)$. Global developmental delay became apparent at 1 year. At 15 months he was able to turn from supine to prone. Clinical features including ataxia, repeatedly pathological EEG, and a cheerful manner suggested Angelman syndrome. No speech development was apparent. At the age of 5 years, he was able to crawl and to walk with assistance. His weight was $15 \mathrm{~kg}\left(<3^{\text {rd }}\right.$ centile, -2.1 S.D. $)$, his height $97 \mathrm{~cm}\left(<3^{\text {rd }}\right.$ centile, -3.1 S.D. $)$, and his head circumference $48 \mathrm{~cm}\left(<3^{\text {rd }}\right.$ centile, -2.9 S.D. $)$. He showed slight dysmorphisms such as a prominent forehead, arched eyebrows, slightly upslanting palpebral fissures, smooth philtrum, thin upper lip, and widely-spaced teeth, but no major dysmorphic stigmata (Fig. 1d).

Conventional karyotyping, subtelomere screening, methylation analysis at the 15q11.2 locus, and sequencing of $U B E 3 A$ and SLC9A6 gave normal results.

\section{Patient 3}

Patient 3 is a 6-year-old girl born as the first child of a French mother and an unknown father. Her 3-year-old maternal half-brother shows normal development. The mother, who was 15 years old at the birth of her daughter, has mild intellectual disability and poor language abilities. She has normal measurements without micro- 

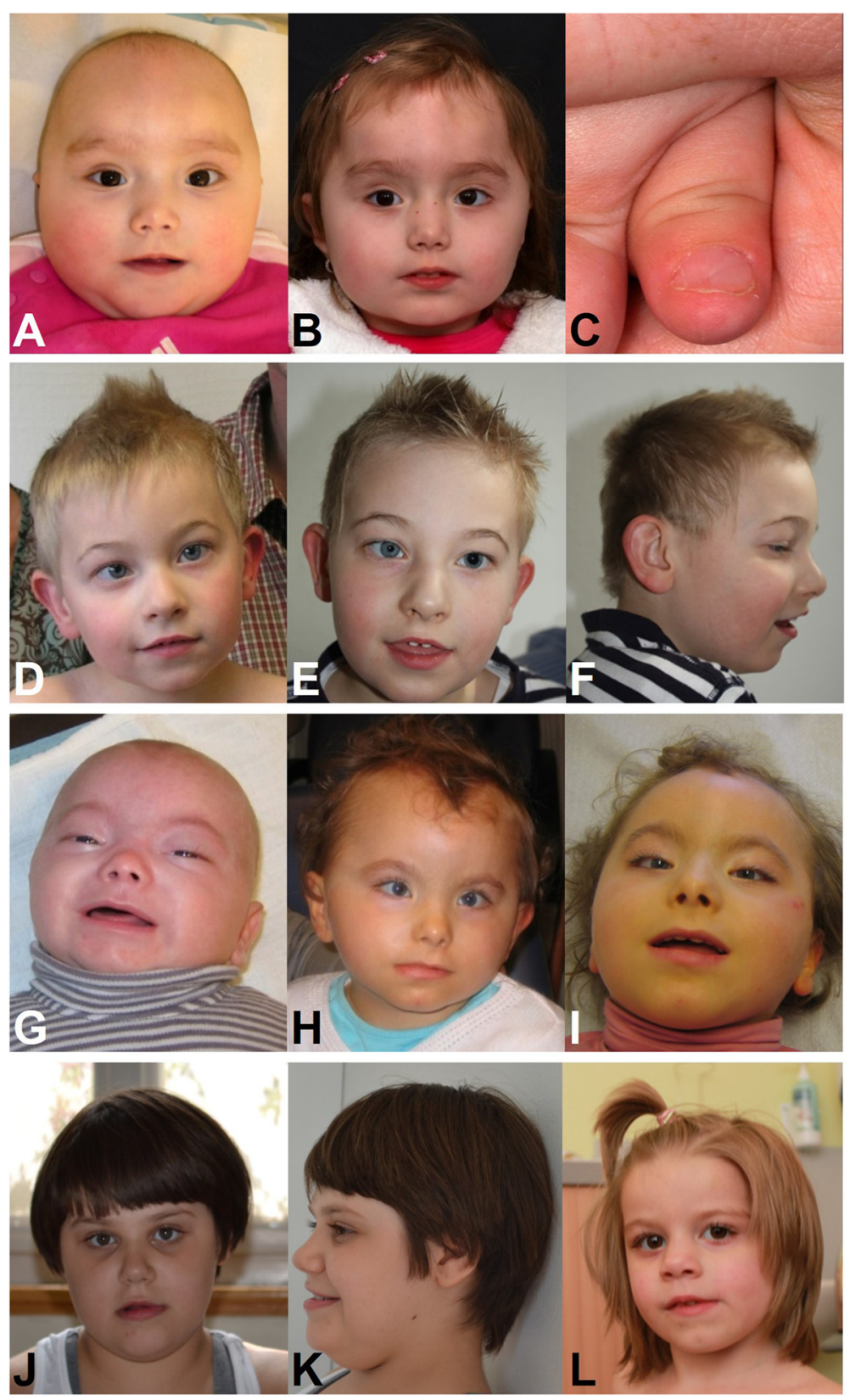

Fig. 1 Facial phenotypes and nail abnormalities of patients with 9q33.3-q34.1 deletion. Facial phenotypes of patients 1-5 (a, b and $\mathbf{d}-\mathbf{l})$ and nail abnormalities of patient 1 (c). Patient 1 at the age of 11 months (a) and 35 months (b). Note round face, telecanthus, upslanting palpebral fissures, epicanthic folds, short nose, thin upper lip, low-set ears, tapering fingers, and ridged nail of the thumb (c). Patient 2 at the age of 5 years (d), and 6 years (e, f). Note prominent forehead, arched eyebrows, slightly upslanting palpebral fissures, and thin upper lip. Patient 3 at the age of 4 years (I). Note high frontal hairline, a short nose with anteverted nares and a dimpled chin. Patient 4 at the age of 5 months ( $(\mathbf{g}), 3$ years (h), and 5 years (i). Note high frontal hairline, telecanthus, upslanting palpebral fissures, arched eyebrows, and thin upper lip. Patient 5 at the age of 11 years (j, k). Note round face, thin upper lip, and prominent lower lip

or brachycephaly and no seizure history. Her only dysmorphisms are mildly anteverted nares and a dimpled chin.

Patient 3 was born at gestational week 35 with a weight of $2630 \mathrm{~g}$ ( $50^{\text {th }}$ centile), a length of $45 \mathrm{~cm}\left(25^{\text {th }}\right.$ centile, -1.0 S.D.), and an OFC of $31 \mathrm{~cm}\left(10^{\text {th }}\right.$ centile, -1.5 S.D.). Mild muscular hypotonia was noted as well as talus valgus. She started walking at the age of 14 months and walked without support at 22 months. Neither feeding difficulties nor obesity were reported in the first 2 years of life. There was no active speech development. Her recurrent otitis was treated repeatedly with transtympanic drains. 
Upon clinical genetic examination at the age of 4 years and 5 months, her weight was $13 \mathrm{~kg}$ ( $3^{\text {rd }}$ centile, -2.0 S.D.), her height was $92 \mathrm{~cm}$ ( $3^{\text {rd }}$ centile, -2.0 S.D.), and her OFC was $45.5 \mathrm{~cm}$ ( $3^{\text {rd }}$ centile, -2.0 S.D.). She was microcephalic but not brachycephalic. A high frontal hairline, convergent strabismus, a short nose with anteverted nares and a dimpled chin were noted (Fig. 11). Hands and fingers were normal. Language development was severely delayed with only few syllables and cries as vocalizations. She showed stereotypic hand movements and autistiform behavior. She suffered from seizures which were treated with Valproate. A cerebral MRI scan showed bilateral temporal-occipital pachygyria with heterogeneous "nodules" of grey cortex. Upon examination at the age of 6 years, her weight was $19.4 \mathrm{~kg}\left(25^{\text {th }}\right.$ centile,

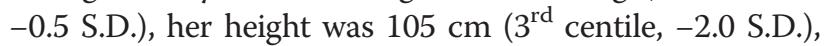
and her OFC remained at $48 \mathrm{~cm}\left(3^{\text {rd }}\right.$ centile, -2.0 S.D.). Severe intellectual disability, muscular hypotonia, and autistic behavior were still present. She showed bruxism and her language was limited to repeating single words. She had strabismus, a short nose with anteverted nares, and a flat face, as well as long and thin fingers and pes planus. At the age of 9 years, she was still reported to have no active speech and to have remained seizure-free under treatment for 20 months.

FMR1 analyses by PCR and Southern blot and MECP2 analysis (DHPLC) gave normal results.

\section{Patient 4}

Patient 4 is a 5 year and 6 months old girl born prematurely after 27 weeks of gestation because of a placental hematoma. Her unrelated parents are of French descent and there is no particular familial history of genetic disease. Birth weight was $950 \mathrm{~g}\left(65^{\text {th }}\right.$ centile, +0.5 S.D., agecorrected), height was $34 \mathrm{~cm}$ ( $37^{\text {th }}$ centile, -0.5 S.D.), and OFC $24 \mathrm{~cm}\left(29^{\text {th }}\right.$ centile, -0.5 S.D.). Bilateral talipes equinus and a patent ductus arteriosus which required surgical treatment were noted at birth. She had retinopathy of prematurity with vitreous hemorrhage as well as bilateral microphtalmia and alternate strabismus. At the age of 2 months, she presented with generalized seizures with persistent, almost daily crises which were resistant to treatment. A brain MRI gave normal results. Arm and forearm X-rays showed diffuse bone demineralization and radiocubital synostosis.

She was first referred for clinical genetic evaluation at the age of 5 months. Dysmorphic features including high frontal hairline, telecanthus, upslanting palpebral fissures, arched eyebrows, flat nasal bridge, thin upper lip, and retrognathism were noted (Fig. 1g). She also had bilateral 5th finger clinodactyly, absent thumb nails, and arched and abnormal nails of both index fingers. Her weight was $3625 \mathrm{~g}$ ( $5^{\text {th }}$ centile, -1.9 S.D., age-corrected), height $49.5 \mathrm{~cm} \mathrm{(1 \textrm {st }}$ centile, -2.5 S.D., age-corrected), and
OFC $35 \mathrm{~cm}\left(<3^{\text {rd }}\right.$ centile, -3.2 S.D. $)$. Standard karyotyping gave normal results. At last examination at the age of 5 years and 6 months (Fig. 1i), she still had developed no active language but showed eye contact and was able to smile. Sitting without support had not been acquired. Her weight was $16 \mathrm{~kg}$ ( $8^{\text {th }}$ centile, -1.8 S.D.), height $96 \mathrm{~cm}$ (below the $1^{\text {st }}$ centile, -3.5 S.D.), and her OFC $47 \mathrm{~cm}\left(2^{\text {nd }}\right.$ centile, -2.2 S.D.). Nephropathy was diagnosed and CT scans at the age of 5 years and 6 months showed non-ossified and dislocated patellae, with bilateral patellar tendon hypoplasia.

\section{Patient 5}

Patient 5 was published previously without clinical detail [6]. She was the first child to non-consanguineous healthy Brazilian-Swiss parents after eight miscarriages and two unsuccessful IVF attempts. The family history was otherwise unremarkable. After an uneventful pregnancy, the girl was born at term by Cesarean section. At birth, her weight was $2500 \mathrm{~g}\left(3^{\text {rd }}-10^{\text {th }}\right.$ centile, -2.0 S.D. $)$ and her length $48 \mathrm{~cm}\left(25-50^{\text {th }}\right.$ centile, -1.2 S.D.); her head circumference was not documented.

As a neonate she presented with muscular hypotonia and subsequently with pronounced early developmental delay. She started sitting without support at 1 year of age and started walking at 4 years of age. She developed truncal and gait ataxia. Cerebral MRI and EEG exams at 6 years of age gave normal results. She never presented with clinical seizures. Sleeping issues were apparent, including trouble in falling asleep or to sleep through the night, and parasomnia with episodes of crying and screaming. Polysomnography at 10 years of age did not show EEG anomalies but confirmed episodes of arousal and pavor nocturnus. At the age of 11 years and 7 months, she spoke a few words. Auditory testing was normal. Except for strabismus, there were no visual impairments. Celiac disease was confirmed by biopsy and treated by diet. Toilet training has never been achieved.

At 11 years and 7 months of age, the patient presented with truncal obesity (OFC $53 \mathrm{~cm}, 25-50^{\text {th }}$ centile, -1.2 S.D.; length $137 \mathrm{~cm}, 10^{\text {th }}$ centile, -1.5 S.D.; weight $44 \mathrm{~kg}, 50-75^{\mathrm{th}}$ centile, +0.6 S.D.). She was restless and hyperactive, limiting the overall physical exam. She did not have specific dysmorphic signs; a round face, round eyes with long eyelashes, strabismus, a short nose, a thin upper lip, and an everted lower lip were noted (Fig. 1j, k). Hands and feet were small with pes planus. Hypotonia and ataxia were evident.

Previous genetic testing for Angelman syndrome (methylation specific PCR) and karyotype analysis as well as standard metabolic testing results gave normal results. 


\section{Chromosomal micro-array analyses}

In patients 1, 2, 4 and 5, de novo deletions in 9q33.3-q34.11 ranging from 1.23 to $4.13 \mathrm{Mb}$ were detected using various array platforms (Table 1, Fig. 2). Interestingly, the smallest deletion was detected in patient 3 , whose $432-\mathrm{kb}$ deletion was inherited from her mother who is reported to have mild ID. The smallest region of overlap (SRO) is defined by the distal boundary of the deletion in patient 3 and the proximal deletion boundary in patient 5 , and includes only two RefSeq genes, RALGPS1 and GARNL3. In patients 1, 2, 3 and 4, ANGPTL2 is also deleted. Importantly, the SRO does not include the STXBP1 gene in 9q34.11 (Fig. 2). No additional de novo CNVs or CNVs in known copy numbersensitive regions have been detected.

\section{Sanger sequencing of SRO genes RALGPS1 and GARNL3}

Every exon including exon/intron boundaries of all known protein-coding RefSeq isoforms of GARNL3 and RALGPS1 has been studied in at least 156 of the 192 ID/DD patients. The sequencing revealed eight rare variants in GARNL3 and none in RALGPS1 (Additional file 1: Table S1). All GARNL3 variants were present in a heterozygous state in either one or two patients with ID/DD (minor allele frequencies $/ \mathrm{MAF}<0.58 \%$ ). Four variants caused amino acid changes whereas the remaining four variants were synonymous. In total, four of the eight GARNL3 variants were listed in dbSNP build 134 and were also present in the NHLBI GO-ESP cohort (Exome Variant Server, NHLBI GO Exome Sequencing Project (ESP), http://evs.gs.washington. edu/EVS/; July 2015). The remaining four variants were followed up by pedigree analyses. All variants had been inherited from an unaffected parent (Additional file 1: Table S1), suggesting no obvious association between the variants and the occurrence of ID.

\section{Expression analyses of SRO genes and STXBP1}

We performed gene expression studies with predesigned Taqman gene expression assays for GARNL3, RALGPS1, STXBP1, and ANGPTL2 on whole blood RNA of five patients with microdeletion and of five unaffected control persons. The expression level of ANGPTL2 which is not contained in the SRO but in four of the detected microdeletions including the smallest one was determined to be too low in whole blood RNA to obtain results.

Compared to the mean of five unaffected controls, the expression levels of GARNL3 were significantly $(p<$ $0.05)$ reduced in all five patients (Fig. 3a). For RALGPS1, the expression was reduced significantly in patients 1 through 4, but not in patient 5 (Fig. 3b). STXBP1 showed a significantly reduced expression in patients 1 , 2 , and 4, while its expression in patients 3 and 5 was not reduced significantly (Fig. 3c).

\section{Discussion}

Array CGH is now widely used in cytogenetics centers for postnatal constitutional genome analysis and in research for the identification of new causative microdeletion and microduplication syndromes or copy number variations (CNVs) and has alos been instrumental in the identification of new candidate genes for e.g. intellectual disability (ID) [7-9].

Several microdeletions involving the chromosomal region 9q33.3-q34.11 have been reported and seem to be associated with a range of clinical phenotypes including, but not limited to, ID, developmental delay (DD), and seizures $[10,11]$. Several studies identifying such microdeletions had in fact been focused on patients with diverse seizure phenotypes $[1,12]$ or aimed at identifying mutations including CNVs involving STXBP1. De novo missense, nonsense, frameshift, and splice-site mutations, as well as genomic deletions of STXBP1, have been found in association with Early Infantile Epileptic Encephalopathy 4 (EIEE4; OMIM \#612164) and other epilepsy types [13], such that STXBP1 haploinsufficiency is the accepted cause of these epileptic phenotypes. Consequently, all the published microdeletions of 9q33.3q34.11 contained STXBP1 and all published microdeletion carriers suffered from seizures.

Here, we report five novel patients with microdeletions of $9 \mathrm{q} 33.3$ only or 9q33.3-q34.11 which vary greatly in size (0.4-4.1 Mb; Table 1) and gene content (3-95 RefSeq genes). The smallest deletion reported here encompasses only three RefSeq genes (RALGPS1, ANGPTL2, and GARNL3). In spite of the differences between the breakpoints of their deletions, the patients show considerable clinical similarities. All five patients have ID and DD with pronounced speech delay or impairment, muscular hypotonia, and strabismus. Microcephaly and postnatal (borderline) short stature are present in four of the patients, while only two of them display seizure phenotypes. Other clinical findings shared by at least two patients include ataxia phenotypes (patients 2 and 5), recurrent otitis media (patients 1 and 3), and unspecific brain MRI findings (patients 1, 2, and 3) including delayed myelination in two patients. Common dysmorphisms include a round face (patients 1 and 5), upslanting palpebral fissures (patients 1 and 2), a short nose (patients 1, 2, and 5), and a thin upper lip (patients 1, 2, and 5).

In addition, two of the patients reported here also display typical findings of Nail-Patella syndrome (NPS, OMIM \# 161200), including talipe equinus, reduced pain sensation, high frontal hairline, and ridged nails in patient 1 (Fig. 1c) and bilateral 5th finger clinodactyly, missing nails of the first fingers, talipe equinus, non-ossified and dislocated patellae, and typical forearm malformations in patient 3. However, the gene known to be responsible for NPS, $L M X 1 B$, only appears to be deleted in patient 4 , 
Table 1 Main clinical findings in patients with microdeletions of 9q33.3-q34.1

\begin{tabular}{|c|c|c|c|c|c|c|c|c|c|c|c|}
\hline & & Patient 1 & Patient 2 & Patient 3 & Patient 4 & Patient 5 & $\begin{array}{l}\text { Campbell et al. } \\
2012[11] \\
\text { (patient 3) }\end{array}$ & $\begin{array}{l}\text { Campbell et al. } \\
2012[11] \\
\text { (patient 10) }\end{array}$ & $\begin{array}{l}\text { Saitsu et al. } \\
2008 \text { [1] } \\
\text { (patient 1) }\end{array}$ & $\begin{array}{l}\text { Saitsu et al. } \\
2012[12] \\
\text { (Patient 2231 a })\end{array}$ & $\begin{array}{l}\text { Mignot et al. } \\
2011 \text { [13] } \\
\text { (patient 3) }\end{array}$ \\
\hline Deletion size & & $1.76 \mathrm{Mb}$ & $1.20 \mathrm{Mb}$ & $432 \mathrm{~kb}$ & $4.13 \mathrm{Mb}$ & $1.23 \mathrm{Mb}$ & $2.65 \mathrm{Mb}$ & $830 \mathrm{~kb}$ & $2.10 \mathrm{Mb}$ & $2.85 \mathrm{Mb}$ & $3.3 \mathrm{Mb}$ \\
\hline ISCN 2013 & & $\begin{array}{l}\text { arr [hg19] } \\
9 q 33.3 q 34.11 \\
(129,463,613- \\
131,224,189) \\
\times 1\end{array}$ & $\begin{array}{l}\text { arr [hg19] } \\
9 q 33.3 q 34.11 \\
(129,630,976- \\
130,833,333) \\
\times 1\end{array}$ & $\begin{array}{l}\text { arr [hg19] } \\
9933.3 \\
(129,688,382- \\
130,120,163) \\
\times 1\end{array}$ & $\begin{array}{l}\text { arr [hg19] } \\
9 q 33.3 q 34.11 \\
(128,870,221- \\
132,995,660) \\
\times 1\end{array}$ & $\begin{array}{l}\text { arr [hg19] } \\
9 q 33.3 q 34.11 \\
(129,950,179- \\
131,180,179) \\
\times 1\end{array}$ & $\begin{array}{l}\text { arr [hg19] } \\
9 q 33.3 q 34.11 \\
(129,473,714- \\
131,633,299) \\
\times 1\end{array}$ & $\begin{array}{l}\text { arr [hg19] } \\
9 q 33.3 q 34.11 \\
(129,843,508- \\
130,681,956) \\
\times 1\end{array}$ & $\begin{array}{l}\text { arr [hg19] } \\
9 q 33.3 q 34.11 \\
(129,060,488- \\
131,199,630) \\
\times 1\end{array}$ & $\begin{array}{l}\text { arr [hg19] } \\
9 q 33.3 q 34.11 \\
(129,980,488- \\
132,830,579) \\
\times 1\end{array}$ & $\begin{array}{l}\text { n.r. del } \\
9 \text { q33.3-q34.11 }\end{array}$ \\
\hline Affected genes & & $\begin{array}{l}\text { RALGPS1, } \\
\text { ANGPTL2, } \\
\text { GARNL3 + 48 } \\
\text { RefSeq genes } \\
\text { incl. STXBP1 }\end{array}$ & $\begin{array}{l}\text { RALGPS1, } \\
\text { ANGPTL2, } \\
\text { GARNL3 }+28 \\
\text { RefSeq genes } \\
\text { incl. STXBP1 }\end{array}$ & $\begin{array}{l}\text { RALGPS1, } \\
\text { ANGPTL2, } \\
\text { GARNL3 }\end{array}$ & $\begin{array}{l}\text { RALGPS1, } \\
\text { ANGPTL2, } \\
\text { GARNL3 }+92 \\
\text { RefSeq genes } \\
\text { incl. STXBP1 }\end{array}$ & $\begin{array}{l}\text { RALGPS1, } \\
\text { GARNL3 + } 45 \\
\text { RefSeq genes } \\
\text { incl. STXBP1 }\end{array}$ & $\begin{array}{l}\text { RALGPS1, } \\
\text { ANGPTL2, } \\
\text { GARNL3 }+58 \\
\text { RefSeq genes } \\
\text { incl. STXBP1 }\end{array}$ & $\begin{array}{l}\text { RALGPS1, } \\
\text { ANGPTL2, } \\
\text { GARNL3 }+22 \\
\text { RefSeq genes } \\
\text { incl. STXBP1 }\end{array}$ & $\begin{array}{l}\text { RALGPS1, } \\
\text { ANGPTL2, } \\
\text { GARNL3 }+50 \\
\text { RefSeq genes } \\
\text { incl. STXBP1 }\end{array}$ & $\begin{array}{l}\text { RALGPS1, } \\
\text { GARNL3 + } 86 \\
\text { RefSeq genes } \\
\text { incl. STXBP1 }\end{array}$ & $\begin{array}{l}\text { RALGPS1, } \\
\text { ANGPTL2, } \\
\text { GARNL3 + } \\
>9 \text { RefSeq } \\
\text { genes incl. } \\
\text { STXBP1 }\end{array}$ \\
\hline Origin & & De novo & De novo & Maternal & De novo & De novo & De novo & De novo & De novo & De novo & De novo \\
\hline Age & & $3 y$ & $5 y$ & $6 y$ & $5 \mathrm{y}, 6 \mathrm{mo}$ & $11 \mathrm{y}, 7 \mathrm{mo}$ & $6 y$ & $6 y$ & $2 \mathrm{y}, 11 \mathrm{mo}$ & $1 \mathrm{y}, 7 \mathrm{mo}$ & $10 y$ \\
\hline Sex & & Female & Male & Female & Female & Female & male & Male & Female & Male & Female \\
\hline \multirow[t]{3}{*}{$\begin{array}{l}\text { Birth } \\
\text { parameters }\end{array}$} & Length & $<10^{\text {th }}$ centile & $<3^{\text {rd }}$ centile & $25^{\text {th }}$ centile & $10^{\text {th }}$ centile & ND & n.r. & n.r. & $-2.3 \mathrm{SD}$ & Normal & $\begin{array}{l}\sim 10^{\text {th }} \text { centile } \\
\text { (est.) }\end{array}$ \\
\hline & Weight & $10^{\text {th }}$ centile & $3^{\text {rd }}$ centile & $50^{\text {th }}$ centile & $10^{\text {th }}$ centile & $\begin{array}{l}0.4^{\text {th }}-2^{\text {nd }} \\
\text { centile }\end{array}$ & n.r. & n.r. & $-2.4 \mathrm{SD}$ & Normal & $\begin{array}{l}\sim 10^{\text {th }} \text { centile } \\
\text { (est.) }\end{array}$ \\
\hline & OFC & $<3^{\text {rd }}$ centile & $<3^{\text {rd }}$ centile & $10^{\text {th }}$ centile & $10^{\text {th }}$ centile & ND & n.r. & n.r. & $-0.8 \mathrm{SD}$ & Normal & ND \\
\hline \multirow{3}{*}{$\begin{array}{l}\text { Growth } \\
\text { parameters } \\
\text { (at examination) }\end{array}$} & Height & $10^{\text {th }}$ centile & $\begin{array}{l}4.5 \mathrm{~cm}<3^{\text {rd }} \\
\text { centile }\end{array}$ & $\begin{array}{l}\sim 6 \mathrm{~cm} \\
<3^{\text {rd }} \text { centile }\end{array}$ & $3^{\text {rd }}$ centile & $0.4^{\text {th }}$ centile & n.r. & n.r. & $<-2 S D^{a}$ & n.r. & $10^{\text {th }}$ centile \\
\hline & Weight & $25-50^{\text {th }}$ centile & $10^{\text {th }}$ centile & $10-25^{\text {th }}$ centile & $10^{\text {th }}$ centile & $25^{\text {th }}$ centile & n.r. & n.r. & $<-2 S D^{a}$ & n.r. & $\begin{array}{l}75-90^{\text {th }} \\
\text { centile }\end{array}$ \\
\hline & OFC & $\begin{array}{l}\sim 1.5 \mathrm{~cm} \\
<3^{\text {rd }} \text { centile }\end{array}$ & $\begin{array}{l}2 \mathrm{~cm}<3^{\text {rd }} \\
\text { centile }\end{array}$ & $\begin{array}{l}\sim 0.5 \mathrm{~cm}<3^{\text {rd }} \\
\text { centile }\end{array}$ & $10^{\text {th }}$ centile & $<0.4^{\text {th }}$ centile & n.r. & n.r. & $\begin{array}{l}40.0 \mathrm{~cm} \\
\left(<<3^{\text {rd }} \text { centile }\right)^{a}\end{array}$ & $-3 S D$ & $\begin{array}{l}50-75^{\text {th }} \\
\text { centile }\end{array}$ \\
\hline Microcephaly & & + & + & + & - & + & + & n.r.. & n.r. & + & + \\
\hline $\begin{array}{l}\text { Intellectual } \\
\text { disability }\end{array}$ & & + & + & + & + & + & + & + & + & + & + \\
\hline Speech & & $\begin{array}{l}\text { Delayed } \\
\text { (only syllables) }\end{array}$ & No speech & $\begin{array}{l}\text { No speech } \\
\text { (some syllables } \\
\text { and cries) }\end{array}$ & No speech & $\begin{array}{l}\text { Delayed } \\
\text { (few words) }\end{array}$ & $\begin{array}{l}\text { Severely } \\
\text { impaired }\end{array}$ & $\begin{array}{l}\text { Severely } \\
\text { impaired }\end{array}$ & No speech & n.r. & $\begin{array}{l}\text { Delayed } \\
\text { (few words) }\end{array}$ \\
\hline Walking & & $\begin{array}{l}\text { No (no } \\
\text { crawling/ } \\
\text { sitting/ } \\
\text { standing) }\end{array}$ & $\begin{array}{l}\text { Yes (only with } \\
\text { assistance) }\end{array}$ & $\begin{array}{l}\text { Yes (walking } \\
\text { age } 22 \text { mo) }\end{array}$ & No & $\begin{array}{l}\text { Yes (walking } \\
\text { age } 4 \text { y) }\end{array}$ & n.r. & n.r. & No & n.r. & No \\
\hline
\end{tabular}


Table 1 Main clinical findings in patients with microdeletions of 9q33.3-q34.1 (Continued)

\begin{tabular}{|c|c|c|c|c|c|c|c|c|c|c|}
\hline Seizures & - & - & + & + & - & + & - & + & + & + \\
\hline EEG & Pathologic & Pathologic & Normal & Pathologic & Normal & $\begin{array}{l}\text { Right temporal } \\
\text { spikes }\end{array}$ & Normal & $\begin{array}{l}\text { Suppression- } \\
\text { burst pattern }\end{array}$ & $\begin{array}{l}\text { Suppression- } \\
\text { burst pattern }\end{array}$ & $\begin{array}{l}\text { Disorganized } \\
\text { background } \\
\text { activity, } \\
\text { multifocal } \\
\text { spikes, spike } \\
\text { and spike- } \\
\text { wave bursts }\end{array}$ \\
\hline Brain MRI & $\begin{array}{l}\text { Mildly delayed } \\
\text { myelination }\end{array}$ & $\begin{array}{l}\text { Delayed } \\
\text { myelination, } \\
\text { unilateral } \\
\text { temporal } \\
\text { closed lip } \\
\text { schizence-phaly }\end{array}$ & $\begin{array}{l}\text { Bilateral } \\
\text { temporo- } \\
\text { occipital } \\
\text { pachygyria } \\
\text { with } \\
\text { heterogenous } \\
\text { "nodules" of } \\
\text { grey }\end{array}$ & Normal & Normal & $\begin{array}{l}\text { Chiari type I } \\
\text { malformation }\end{array}$ & Normal & $\begin{array}{l}\text { Cortical } \\
\text { atrophy, } \\
\text { diffuse } \\
\text { hypomyeli- } \\
\text { nation, thin } \\
\text { corpus } \\
\text { callosum, } \\
\text { cerebellum } \\
\text { and brain } \\
\text { stem atrophy } \\
\text { (at } 12 \text { mo) }\end{array}$ & $\begin{array}{l}\text { Thin corpus } \\
\text { callosum, } \\
\text { relatively } \\
\text { small } \\
\text { cerebellum } \\
(2 \mathrm{mo})\end{array}$ & $\begin{array}{l}\text { Global } \\
\text { atrophy of } \\
\text { cerebral } \\
\text { hemispheres }\end{array}$ \\
\hline $\begin{array}{l}\text { Muscular } \\
\text { hypotonia }\end{array}$ & + & + & + & + & + & + & + & + & n.r. & n.r. \\
\hline Dysmorphisms & $\begin{array}{l}\text { Round face, } \\
\text { high frontal } \\
\text { hairline, } \\
\text { telecanthus, } \\
\text { upslanting } \\
\text { palpebral } \\
\text { fissures, short } \\
\text { nose with } \\
\text { depressed } \\
\text { bridge, thin } \\
\text { upper lip, low- } \\
\text { set ears with } \\
\text { attached lob } \\
\text { ules, short neck, } \\
\text { tapering fingers } \\
\text { with ridged } \\
\text { nails, talipe } \\
\text { equinus }\end{array}$ & $\begin{array}{l}\text { Arched } \\
\text { eyebrows, } \\
\text { slightly } \\
\text { upslanting } \\
\text { palpebral } \\
\text { fissures, smooth } \\
\text { philtrum, } \\
\text { widely-spaced } \\
\text { teeth, thin } \\
\text { upper lip }\end{array}$ & $\begin{array}{l}\text { Short nose, } \\
\text { anteverted } \\
\text { nares, high } \\
\text { frontal hairline, } \\
\text { dimple chin, } \\
\text { talus valgus } \\
\text { bilateral }\end{array}$ & $\begin{array}{l}\text { Arched } \\
\text { eyebrows, } \\
\text { telecanthus, } \\
\text { horizontal } \\
\text { palpebral } \\
\text { fissures, flat } \\
\text { nasal bridge, } \\
\text { retrognathia, } \\
\text { bilateral 5th } \\
\text { finger } \\
\text { clinodactyly, } \\
\text { 1st fingers: } \\
\text { absent nails, } \\
\text { talipe equinus }\end{array}$ & $\begin{array}{l}\text { Round face, } \\
\text { round eyes, } \\
\text { long eyelashes, } \\
\text { short nose, } \\
\text { thin upper lip, } \\
\text { slightly everted } \\
\text { lower lip, small } \\
\text { hands and feet }\end{array}$ & $\begin{array}{l}\text { Dysmorphic } \\
\text { features }\end{array}$ & $\begin{array}{l}\text { Mild } \\
\text { dysmorphic } \\
\text { features }\end{array}$ & n.r. & $\begin{array}{l}\text { Midface } \\
\text { hypoplasia }^{a}\end{array}$ & $\begin{array}{l}\text { dysmorphic } \\
\text { features }\end{array}$ \\
\hline Ocular findings & Strabismus & $\begin{array}{l}\text { Strabismus, } \\
\text { hypermetropia }\end{array}$ & Strabismus & $\begin{array}{l}\text { Strabismus, } \\
\text { retinopathy } \\
\text { of prematurity, } \\
\text { bilateral } \\
\text { microphtalmia }\end{array}$ & Strabismus & Strabismus & - & n.r. & n.r. & n.r. \\
\hline Behavior & Bruxism & $\begin{array}{l}\text { Cheerful } \\
\text { mannerism }\end{array}$ & $\begin{array}{l}\text { Autistic } \\
\text { features, stereotypic } \\
\text { hand } \\
\text { movements }\end{array}$ & ND & $\begin{array}{l}\text { Hyperactive, } \\
\text { sleep } \\
\text { difficulties }\end{array}$ & n.r. & n.r. & n.r. & n.r. & Stereotypies \\
\hline
\end{tabular}


Table 1 Main clinical findings in patients with microdeletions of 9q33.3-q34.1 (Continued)

\begin{tabular}{|c|c|c|c|c|c|c|c|c|c|}
\hline Other findings & $\begin{array}{l}\text { Recurrent otitis } \\
\text { media, lack of } \\
\text { pain sensation }\end{array}$ & $\begin{array}{l}\text { Hypotonic- } \\
\text { ataxic } \\
\text { movement } \\
\text { disorder }\end{array}$ & $\begin{array}{l}\text { Recurrent otitis } \\
\text { media, failure } \\
\text { to thrive }\end{array}$ & $\begin{array}{l}\text { Forearm } \\
\text { malformation, } \\
\text { non-ossified } \\
\text { and } \\
\text { dislocated } \\
\text { patellae, } \\
\text { nephropathy }\end{array}$ & $\begin{array}{l}\text { Trunk and gait } \\
\text { ataxia, pes } \\
\text { planovalgus, } \\
\text { celiac disease, } \\
\text { overweight }\end{array}$ & Ataxia & $\begin{array}{l}\text { Spastic } \\
\text { quadriplegia }\end{array}$ & $\begin{array}{l}\text { Cleft lip/ } \\
\text { palate, } \\
\text { ventricular } \\
\text { septal defect, } \\
\text { overlapping } \\
\text { fingers, small } \\
\text { penis, spastic } \\
\text { quadriplegia, } \\
\text { multiple } \\
\text { arthrogryposis }\end{array}$ & $\begin{array}{l}\text { Absent } \\
\text { thumbnails, } \\
\text { nails of 2nd } \\
\text { fingers } \\
\text { hypoplastic }\end{array}$ \\
\hline
\end{tabular}

Summary of patient phenotypes and deletions

est. estimated, ND not determined, n.r. not reported, OFC occipitofrontal circumference, SD standard deviation, + present, - absent, y years, mo months aPersonal communication. Recurrent dysmorphisms and ocular findings are italicized 


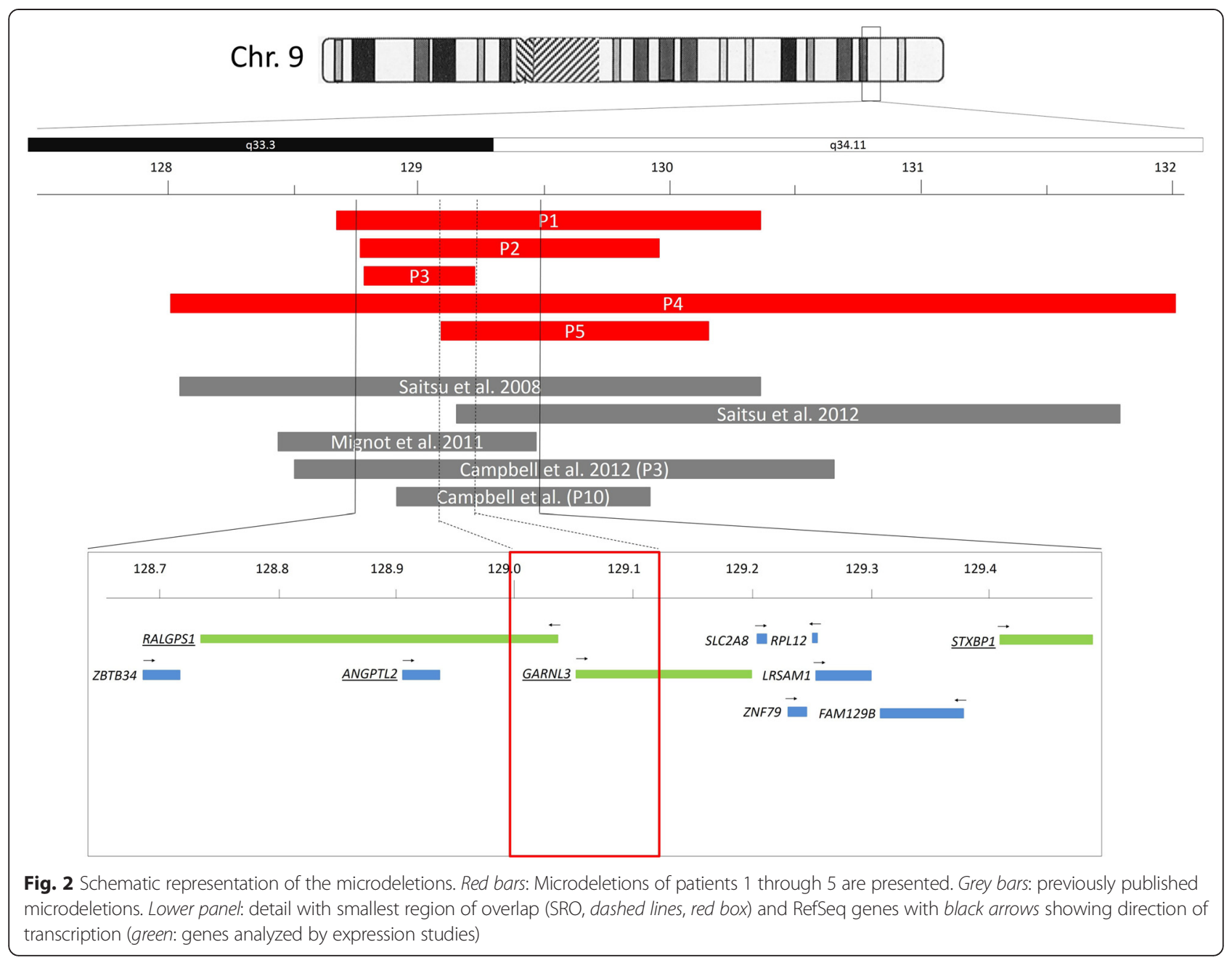

while the minimal deletion interval in patient 1 starts 302 base pairs downstream from $L M X 1 B$. However, as the deletion breakpoints have not been sequenced, it is possible that the deletion in patient 1 in fact could extend into the coding region of $L M X 1 B$, causing features of NPS.

A comparison of the clinical picture of the patients with microdeletions reported here with published carriers of STXBP1 loss-of-function point mutations or intragenic deletions yields several similarities. ID and DD with language delay, muscular hypotonia, seizures, ataxia, and brain MRI findings including hypomyelination were also present in patients with STXBP1 mutations [3-5, 14]. The reduced penetrance regarding seizures reported by Hamdan and colleagues is confirmed and even emphasized by our findings, since only one out of four carriers with STXBP1-encompassing microdeletions reported here suffered from seizures. In contrast to these commonalities, several clinical findings are exclusively present in our patients with microdeletions. To our knowledge, microcephaly, short stature, and strabismus have not been reported in carriers of STXBP1 point mutations or of deletions affecting STXBP1 only. This may also be true for at least some of our patients' dysmorphisms, but in most studies of STXBP1 point mutations, the presence or absence of dysmorphisms is not mentioned. Only Hamdan and colleagues explicitly state that there were no dysmorphic features present in their three patients with truncating STXBP1 mutations. Thus, it may be assumed that the common findings-microcephaly, short stature, and possibly strabismus-which are exclusive to the five patients with microdeletions reported here are unrelated to STXBP1 and associated instead with the smallest region of overlap (SRO) of their deletions. Importantly, our patient 3 presents with seizures although her deletion does not include $S T X B P 1$ and her STXBP1 expression is not reduced. We thus hypothesize that her seizures are unrelated to STXBP1 and may instead be caused by the deletion of the SRO.

We are aware of five previously published patients with microdeletions including these SRO genes (Table 1). For three of these patients, seizures were the primary indication for referral $[1,12,13]$, while two of the patients were referred for array $\mathrm{CGH}$ for a diverse set of clinical 

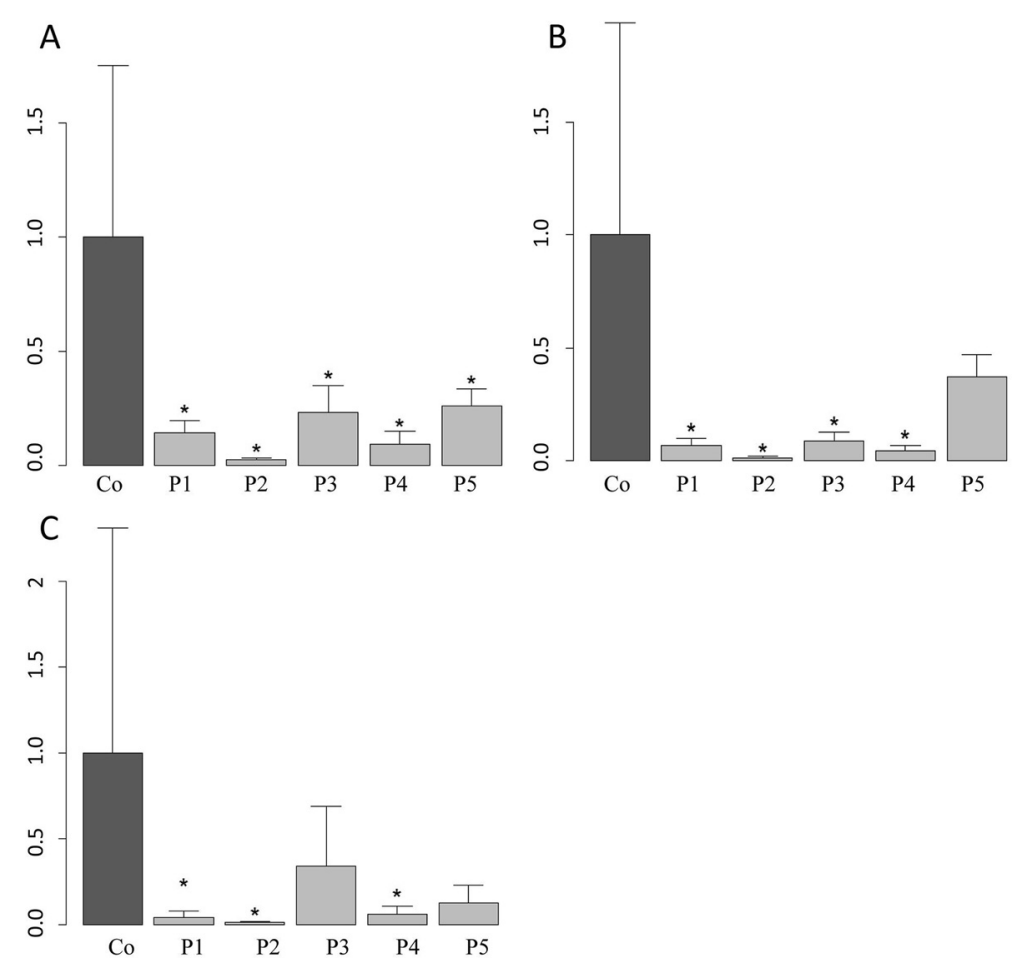

Fig. 3 Results of expression analyses for a GARNL3, b RALGPS1, and c STXBP1. The mean expression of five unaffected controls is set to 1.0 (dark grey bar). The expression level of the patients (light grey) is set in relation to the mean of the five controls. Error bars are based on the data obtained over three experiments. Asterisks mark significant expression differences between patients and controls $(p<0.05$, Wilcoxon-test)

indications [11]. All patients with corresponding clinical data displayed ID and DD with severe language delay and muscular hypotonia. Eight out of nine patients had postnatal microcephaly and five out of seven postnatal short stature or borderline short stature. Seven out of ten patients had brain MRI abnormalities and six out of ten suffered from seizures. When excluding the three patients investigated primarily because of their seizures, to minimize any ascertainment bias, only three out of seven patients suffered from seizures. Dysmorphisms are present in most patients and some dysmorphic features were seen in up to three of the patients presented here. However, there does not seem to be a readily recognizable dysmorphism pattern.

The SRO is localized in distal 9q33.3 and only includes two RefSeq genes, RALGPS1 and GARNL3. In patients 1 through 4, the ANGPTL2 gene is also deleted (Fig. 2). The clinical and genomic evidence may point to the existence of a 9q33.3 microdeletion syndrome which may be unrelated to STXBP1 haploinsufficiency. Until now, none of the three genes has been associated with disease. RALGPS1 (Ral GEF with PH domain and SH3 binding motif 1), formerly known as RalGEF2, encodes a guanine nucleotide exchange factor or GEF for Ral (de Bruyn et al. 2000 [15]). Ral (RalA and RalB) is a small GTPase of the Ras family implicated in the control of cell proliferation, differentiation, cytoskeletal organization, and vesicular transport. One of the mechanisms of Ral activation is the direct binding of active Ras to Ral-specific GEFs such as RALGPS1 [15]. RALGPS1 expression is rather ubiquitous with the highest expression levels in brain, heart, kidney, adrenal gland, and colon. The evidence for RALGPS1 haploinsufficiency is equivocal: the Residual Variation Intolerance (RVI) score, which quantifies gene intolerance to functional mutations [16] is intermediate $(-0.293801652$, corresponding to the 32.9th percentile). As a comparison, the average RVI score for known developmental disorders is lower $(0.56 ; 19.54$ th percentile). On the other hand, its low haploinsufficiency score (HI) of $8.9 \%$ indicates a high probability for haploinsufficiency being pathogenic [17].

Very little is known about the GARNL3 (Homo sapiens GTPase activating Rap/RanGAP domain-like 3) gene. It appears to be involved in the regulation of small GTPasemediated signal transduction and human GARNL3 is rather ubiquitously expressed with the highest expression in brain [18]. Rap/ran-GAP domains are found in GTPase activating proteins (GAP) responsible for the activation of nuclear Ras-related regulatory proteins Rap1, Rsr1 and Ran. Again, the evidence for GARNL3 haploinsufficiency is equivocal with a very low RVI score of -1.082044518 (7.2th percentile) and a rather high haploinsufficiency score (HI) of $33.6 \%$. 
ANGPTL2 (Homo sapiens angiopoietin-like 2, also known as ARP2 for angiopoietin-related protein-2) is discussed here although it is not contained in the SRO because it is deleted in four out of five microdeletions presented here, including the smallest one. It is a secreted glycoprotein that has been implicated in angiogenesis, inflammation, and atherosclerosis, as well as enhancing the survival of human hematopoietic stem cells [19]. It is a member of the Angiopoietin vascular endothelial growth factor family and is largely specific for vascular endothelium. Angptl2 is expressed in the heart, adipose tissue, stomach, small intestine, colon, ovary, uterus, spleen, striated muscle, and, at lower levels, in other tissues and Angptl2 is secreted by different cell types such as adipocytes, endothelial cells, macrophages, keratinocytes and cancer cells $[19,20]$. In zebrafish embryos, Zangptl2 expression was detected in the yolk sac extension and in posterior spinal cord during early development and shown to diminish gradually as development proceeded [21]. During chick embryogenesis, Angptl2 was detected at E3 in the hindbrain and at E4 in neuroepithelium of the forebrain and hindbrain and also in the heart [22]. As part of the Arp2/3 complex, ANGPTL2 interacts directly with the WAVE (Wiskott-Aldrich syndrome protein family verprolinhomologous protein) complex to induce actin cytoskeleton changes and is involved in the regulation of axon growth [23]. Regarding haploinsufficiency prediction, both the RVI score of -0.867014353 (10.7th percentile) and a low HI score of $6.9 \%$ point to ANGPTL2 haploinsufficiency being pathogenic.

In contrast to contiguous gene syndromes such as Williams syndrome, several other microdeletion syndromes have been shown recently to be caused mainly by haploinsufficiency of a single responsible gene such as $M E F 2 C$ in $5 \mathrm{q} 14.3$ microdeletion syndrome [24] or SHANK3 in Phelan-McDermid syndrome [25]. We were interested to determine which scenario applies to microdeletion 9q33.3 syndrome. Overall, neither expression patterns nor functional data nor the RVI and HI scores pointed to one of the SRO genes as the main or even single responsible gene for the 9q33.3-q34.1 microdeletions reported here. Consequently, we analyzed the expression levels of RALGPS1, GARNL3, and ANGPTL2 to gain possible evidence for their haploinsufficiency. We included STXBP1 in our expression studies in order to investigate the possibility of a reduced expression caused by long-range position effects of the deletion in patient 3 which did not include STXBP1. The gene expression studies for GARNL3, RALGPS1, ANGPTL2, and $S T X B P 1$ on whole blood RNA of patients 1 through 5 demonstrated significantly reduced expression levels of the SRO gene GARNL3 in all patients. The expression level of the non-SRO gene ANGPTL2 in whole blood
RNA was too low to be analyzed. RALGPS1 expression was reduced significantly in patients 1 through 4 but not in patient 5 whose deletion did not encompass the gene. STXBP1 did not show a significantly reduced expression in patient 3, in whom STXBP1 was not deleted, thus excluding a possible long-range position effect for her deletion. Our expression studies did not narrow down the causative genes by providing evidence for haploinsufficiency of only one of the SRO genes. Thus, we performed Sanger sequencing of the SRO genes RALGPS1 and GARNL3 in more than 156 patients with ID and DD in order to find possible evidence of loss-of-function mutations in one of these genes as a frequent cause for ID and DD. However, all detected variants were either known single nucleotide polymorphisms listed in dbSNP or had been inherited from an unaffected parent. Thus, no causative mutations were detected and deleterious sequence mutations in RALGPS1 or GARNL3 are apparently not a common cause of ID and DD.

\section{Conclusions}

In summary, we present evidence for a novel $9 \mathrm{q} 33.3$ microdeletion syndrome consisting of ID, DD with pronounced speech delay, muscular hypotonia, strabismus, and incompletely penetrant microcephaly, short stature, and seizures. The smallest region of deletion overlap affects only two coding genes, RALGPS1 and GARNL3 and is localized in close proximity to the known ID / epilepsy gene STXBP1. The seizure, ID, and DD phenotype in the deletions described here is at least partly independent from $S T X B P 1$, as shown by microdeletion sizes and expression studies. Follow-up studies on additional patients are needed to determine if this syndrome is caused by the deletion of only one of the candidate genes RALGPS1 and GARNL3, or if it constitutes a contiguous gene syndrome.

\section{Methods \\ Clinical reports}

Investigations were performed in accordance with the protocols of the Declaration of Helsinki and were approved by the Ethikkommission der Medizinischen Fakultät der Universität Bonn (lfd. Nr. 120/99, 131/08). Consent to participate was obtained from the parents for all participants. The retrospective reports of the microdeletions which were detected during routine array diagnostics did not require ethics committee approval at the respective institutions. Written consent for the publication of clinical photographs was obtained from the parents for all five patients.

\section{Array studies and verification}

For patient 1, peripheral blood genomic DNA was analyzed using an Illumina HumanOmni1-Quad array according to the manufacturer's instructions (Illumina, 
Inc., San Diego, CA, USA). CNV calling was performed as published previously $[26,27]$. Real-time quantitative PCR with three independent primer pairs was performed to confirm the deletion in the patient and to test for the presence of the deletion in parental DNA as published previously $[26,27]$. Primer sequences are available upon request.

For patient 2 and his parents, peripheral blood genomic DNA was analyzed according to manufacturer's instructions with Affymetrix Genome-Wide Human SNP Arrays 6.0 (Affymetrix, Santa Clara, CA, USA) with an average distance of $1.3 \mathrm{~kb}$ between neighboring probes. Genotypes were called with Affymetrix Genotyping Console Software v3 (GTC) using the Birdseed algorithm with the default calling threshold of 0.5 and a prior size of 10,000 bases in a simultaneous analysis of the patient-parents trio. Interpretation was originally based on Human Genome Build 36 (NCBI).

Peripheral blood genomic DNA from patients 3 and 4 was analyzed using Agilent Human Genome CGH 44B microarrays (Agilent, Santa Clara, CA, USA) according to the manufacturer's protocols. Female genomic DNA (Promega, Madison, WI, USA) was used as reference and quantitative PCR for verification and segregation analyses was performed with QuantiTect SYBR Green PCR Kits (Qiagen, Valencia, CA, USA).

Microarray analysis of genomic DNA from patient 5 was performed using the $720 \mathrm{~K}$ whole genome tiling NimbleGen CGH array (Nimble-Gen'; Roche NimbleGen Inc, Madison, Wisconsin, USA). Tiling array version Human CGH $3 \times 720 \mathrm{~K}$ WG-T v3.0 array which contains 720,000 probes with a median probe spacing of 2509 bp was applied. The effective average resolution is approximately $50 \mathrm{~kb}$ for a required minimum of 10 consecutive aberrant oligonucleotides. Labeling and hybridization of test and reference DNA was performed according to the manufacturer's protocols. Two-color scanning was performed with an MS 200 Scanner (NimbleGen; Roche NimbleGen Inc, Madison, Wisconsin, USA). Microarray images were acquired using the MS 200 software. Data extraction, analysis, and visualization were performed using the Nimble$\mathrm{Scan}^{\mathrm{Tw}}$ version 2.5 and SignalMap ${ }^{\mathrm{Tw}}$ version 1.9 software. Verification in the proband and segregation analyses in the parents were performed by fluorescence-in-situ hybridization (FISH) using the locus-specific BAC clone RP11-356B19 (9q34.11; BlueGnome, Cambridge, UK).

\section{Sanger sequencing of SRO genes RALGPS1 and GARNL3}

Mutational screening of RALGPS1 and GARNL3 by Sanger sequencing was performed on 192 individuals seen at the University Medical Genetics Clinics in Bonn, 96 of whom had mild ID/DD and 96 of whom had moderate to severe ID/DD. This study group consisted of 66 females (34\%) and 126 males (66 \%). A total of 82 individuals showed no or only negligible dysmorphisms
(43\%), while 109 had dysmorphisms (57\%); of the latter group, 40 patients also showed organ malformations (21\%). Standard evaluation consisted of detailed clinical investigation, conventional karyotyping, and the exclusion of clinically recognizable syndromes with known etiology. The presence of Fragile X syndrome had been excluded in almost all patients. Previous molecular karyotyping with Illumina microarrays $(550,610,660 \mathrm{~K}$ or Omni1-Quad) had identified no pathogenic aberrations. The study was approved by the Institutional Review Board of the University Hospital of Bonn, and informed consent was obtained for all participants.

To target the coding regions of RALGPS1 and GARNL3 and flanking intronic sequence (at least $15 \mathrm{bp}$ ), primers were designed using the online program Primer3 (http://primer3.ut.ee/). All known protein-coding RefSeq isoforms were analyzed: NM_014636, NM_001190728, NM_001190729 and NM_001190730 for RALGPS1, NM_032293 and NM_001286779 for GARNL3. Sequencing was performed with either genomic or whole genome amplified DNA (REPLI-g WGA kit, Qiagen, Hilden, Germany) of the 192 patients with ID/DD using standard procedures. Amplicons were sequenced bi-directionally using the BigDye Terminator v3.1 Cycle Sequencing Kit (Applied Biosystems, Foster City, California, USA). The fluorescently labeled fragments were analyzed on a capillary sequencing system (3130XL Genetic Analyzer, Applied Biosystems). Sequences were analyzed using SeqMan II software (DNAStar, Madison, WI, USA). All primer sequences as well as PCR conditions are available upon request. The frequency in control cohorts was analyzed for all detected variants using dbSNP build 142 and the NHLBI GO-ESP cohort (Exome Variant Server, NHLBI GO Exome Sequencing Project (ESP), http://evs.gs.washington.edu/EVS/; July 2015). The in silico tool CADD (Combined Annotation Dependent Depletion, http://cadd.gs.washington.edu/, [28]) was employed to predict pathogenicity.

\section{Expression analyses of RALGPS1, GARNL3, ANGPTL2, and STXBP1}

Expression analyses for RALGPS1, GARNL3, ANGPTL2, and $S T X B P 1$ were performed for microdeletion patients 1 through 5 and five unaffected control persons. RNA of the five patients and the five unaffected controls was extracted from whole blood using the PAXgene Blood system (Qiagen, Hilden, Germany). RNA was reverse transcribed using Superscript II (Invitrogen, Carlsbad, CA, USA). To measure expression levels, predesigned TaqMan $^{\circ}$ gene expression assays (Applied Biosystems, Foster City, CA, USA; Hs01115436_m1; Hs00171912_m1; Hs01060483_m1; Hs01119036_m1) were used. The gene expression analysis was performed in triplicate, with each reaction containing $2 \mu \mathrm{l}$ of cDNA template in a $10 \mu \mathrm{l}$ reaction volume, on a LightCycler $^{\circ} 480$ Real-Time PCR 
System (Roche, Mannheim, Germany). The cycling conditions were as follows: $50{ }^{\circ} \mathrm{C}$ for $2 \mathrm{~min}$, denaturation at $95{ }^{\circ} \mathrm{C}$ for $10 \mathrm{~min}$ followed by 40 cycles at $95{ }^{\circ} \mathrm{C}$ for $15 \mathrm{~s}$, and a combined annealing and extension step at $60{ }^{\circ} \mathrm{C}$ for $60 \mathrm{~s}$. Duplex reactions contained both target (FAM) and endogenous control (VIC) probes and primers. Two different endogenous controls (Human B2M (beta-2-microglobulin) Endogenous Control; Human PPIA (cyclophilin A) Endogenous Control; TaqMan ${ }^{\circ}$ Endogenous Controls, Applied Biosystems, Foster City, CA, USA) were assayed in parallel and in separate duplex reactions. Normalized results were compared to the results of five unaffected controls. Each triplicate experiment was performed twice. P values were calculated using a two-sided Wilcoxon test.

\section{Additional file}

Additional file 1: Table S1. Sequencing results of GARNL3 in 192

patients with ID/DD.

\section{Competing interests}

The authors declare that they have no competing interests.

\begin{abstract}
Authors' contributions
JKE participated in the design of the study, helped to draft the manuscript and carried out the majority of molecular genetic studies. HE conceived of the study, participated in its design and coordination and drafted the manuscript. KC mainly wrote the case report for patient 1 and supervised the clinical genetic work. JB participated in coordinating and drafting the sequencing studies and performed the final sequencing data analysis. EW participated in the clinical genetic characterization of patient 1 and the supervision of the clinical genetic work. JZ participated in the sequencing studies. ER participated in the clinical genetic characterization of patient 2. $M B$ and UG supervised and interpreted the molecular karyotyping of patient 2. UG participated in the clinical genetic characterization of patient 2. EM participated in the clinical genetic characterization of patient 1. SHH participated in the expression studies. ND participated in the sequencing studies. MM-D participated in the clinical genetic characterization of patient 3 and wrote the case report. AB participated in the clinical characterization of patient 2. SSch participated in the clinical genetic characterization of patient 1. EL participated in the clinical genetic characterization of patient 4 and wrote the case report. GP/MG participated in the clinical genetic characterization of patient 4. AdB and GJ participated in the clinical genetic characterization of patient 3. BR and IF performed and interpreted the molecular karyotyping of patient 5. IF and PM clinically characterized patient 5 and IF wrote the case report. AD participated in the clinical genetic characterization of patient 2 and in writing the case report. JA supervised and interpreted the molecular karyotyping of patients 3 and 4. JAL participated in the design of the study and Sanger sequencing analysis, and helped to draft the manuscript. AMZ participated in the design and coordination of the study and participated in the molecular genetic studies. All authors read and approved the final manuscript.
\end{abstract}

\section{Acknowledgments}

We thank the patients and their families for their kind cooperation. The Bonn and Tübingen centers are part of the German Mental Retardation Network MRNET (funded by the German Federal Ministry of Education and Research as part of the National Genome Research Network NGFNplus, grant numbers 01GS08164 and 01GS08162, German Mental Retardation Network). This work was supported by the BONFOR program of the Medical Faculty of the Rheinische Friedrich-Wilhelms-University, Bonn.

\section{Author details}

'Institute of Human Genetics, University of Bonn, Sigmund-Freud-Strasse 25, 53105 Bonn, Germany. ${ }^{2}$ Department of Genomics, Life \& Brain Center, University of Bonn, Bonn, Germany. ${ }^{3}$ Present Address: Center for Human Genetics, Freiburg, Germany. ${ }^{4}$ Institute of Medical Genetics and Applied Genomics, University of Tübingen, Tübingen, Germany. ${ }^{5}$ Present Address: Genetikum, Neu-Ulm, Germany. ${ }^{6}$ Present Address: IMGM Laboratories GmbH, Martinsried, Germany. ${ }^{7}$ Children's Hospital, University of Tübingen, Tübingen, Germany. ${ }^{8}$ MVZ Dr. Eberhard \& Partner, Dortmund, Germany. ${ }^{9}$ Centre de Génétique, CHU d'Amiens, Amiens, France. ${ }^{10}$ Service de génétique, CHU de Caen, Caen, France. ${ }^{11}$ Service de Neurologie Pédiatrique, CHU d'Amiens, Amiens, France. ${ }^{12}$ Medical Genetics, Centre of Laboratory Medicine, Cantonal Hospital Aarau, Aarau, Switzerland. ${ }^{13}$ Medical Genetics, University Hospital Basel, Basel, Switzerland. ${ }^{14}$ Laboratoire de Génétique Médicale, Hôpital Jeanne de Flandre, CHRU de Lille, Lille, France. ${ }^{15}$ Present Address:

Greenwood Genetic Center, Greenwood, SC, USA.

Received: 17 July 2015 Accepted: 22 September 2015

Published online: 29 September 2015

\section{References}

1. Saitsu H, Kato M, Mizuguchi T, Hamada K, Osaka H, Tohyama J, et al. De novo mutations in the gene encoding STXBP1 (MUNC18-1) cause early infantile epileptic encephalopathy. Nat Genet. 2008;40:782-8.

2. Otsuka M, Oguni H, Liang J-S, Ikeda H, Imai K, Hirasawa K, et al. STXBP1 mutations cause not only Ohtahara syndrome but also West syndrome-result of Japanese cohort study. Epilepsia. 2010;51:2449-52.

3. Deprez L, Weckhuysen S, Holmgren P, Suls A, Van Dyck T, Goossens D, et al. Clinical spectrum of early-onset epileptic encephalopathies associated with STXBP1 mutations. Neurology. 2010;75:1159-65.

4. Hamdan FF, Piton A, Gauthier J, Lortie A, Dubeau F, Dobrzeniecka S, et al. De novo STXBP1 mutations in mental retardation and nonsyndromic epilepsy. Ann Neurol. 2009;65:748-53.

5. Hamdan FF, Gauthier J, Dobrzeniecka S, Lortie A, Mottron L, Vanasse M, et al. Intellectual disability without epilepsy associated with STXBP1 disruption. Eur J Hum Genet. 2011;19:607-9.

6. Filges I, Suda L, Weber P, Datta AN, Fischer D, Dill P, et al. High resolution array in the clinical approach to chromosomal phenotypes. Gene. 2012;495:163-9.

7. Ahn JW, Bint S, Bergbaum A, Mann K, Hall RP, Ogilvie CM. Array CGH as a first line diagnostic test in place of karyotyping for postnatal referrals-results from four years' clinical application for over 8700 patients. Mol Cytogenet. 2013;6:16.

8. Lourov IY, Vorsanova SG, Kurinnaia OS, Zelenova MA, Silvanovich AP, Yurov YB. Molecular karyotyping by array CGH in a Russian cohort of children with intellectual disability, autism, epilepsy and congenital anomalies. Mol Cytogenet. 2012;5:46.

9. Weise A, Mrasek K, Klein E, Mulatinho M, Llerena Jr JC, Hardekopf D, et al. Microdeletion and microduplication syndromes. J Histochem Cytochem. 2012;60(5):346-58.

10. Boone PM, Bacino CA, Shaw CA, Eng PA, Hixson PM, Pursley AN, et al. Detection of clinically relevant exonic copy-number changes by array CGH. Hum Mut. 2010;31:1326-42.

11. Campbell IM, Yatsenko SA, Hixson P, Reimschisel T, Thomas M, Wilson W, et al. Novel 9q34.11 gene deletions encompassing combinations of four Mendelian disease genes: STXBP1, SPTAN1, ENG, and TOR1A. Genet Med. 2012;14:868-76.

12. Saitsu H, Kato M, Shimono M, Senju A, Tanabe S, Kimura T, et al. Association of genomic deletions in the STXBP1 gene with Ohtahara syndrome. Clin Genet. 2012;81:399-402.

13. Mignot C, Moutard M-L, Trouillard O, Gourfinkel-An I, Jacquette A, Arveiler B, et al. STXBP1-related encephalopathy presenting as infantile spasms and generalized tremor in three patients. Epilepsia. 2011;52:1820-7.

14. Carvill GL, Weckhuysen S, McMahon JM, Hartmann C, Moller RS, Hjalgrim H, et al. GABRA1 and STXBP1: novel genetic causes of Dravet syndrome. Neurology. 2014;82:1245-53.

15. de Bruyn KM, de Rooij J, Wolthuis RM, Rehmann H, Wesenbeek J, Cool RH, et al. RalGEF2, a pleckstrin homology domain containing guanine nucleotide exchange factor for Ral. J Biol Chem. 2000;275:29761-6.

16. Petrovski S, Wang Q, Heinzen EL, Allen AS, Goldstein DB. Genic intolerance to functional variation and the interpretation of personal genomes. PLoS Genet. 2013;9:e1003709. 
17. Huang N, Lee I, Marcotte EM, Hurles ME. Characterising and predicting haploinsufficiency in the human genome. PLoS Genet. 2010;6:e1001154.

18. Thierry-Mieg D, Thierry-Mieg J. AceView: a comprehensive cDNA-supported gene and transcripts annotation. Genome Biol. 2006;7 Suppl 1:S12.1-4.

19. Farhat N, Mamarbachi AM, Thorin E, Allen BG. Cloning, expression and purification of functionally active human angiopoietin-like protein 2 . SpringerPlus. 2014;3:337.

20. Tabata M, Kadomatsu T, Fukuhara S, Miyata K, Ito Y, Endo M, et al. Angiopoietin-like protein 2 promotes chronic adipose tissue inflammation and obesity-related systemic insulin resistance. Cell Metab. 2009:10:178-88.

21. Kubota Y, Oike Y, Satoh S, Tabata Y, Niikura Y, Morisada T, et al. Isolation and expression patterns of genes for three angiopoietin-like proteins, Angpt11, 2 and 6 in zebrafish. Gene Expr Patterns. 2005;5:679-85.

22. Niki D, Katsu K, Yokouchi Y. Ontogeny of angiopoietin-like protein 1, 2, 3, 4 , 5, and 7 genes during chick embryonic development. Dev Growth Differ. 2009:51:821-32.

23. Tahirovic S, Hellal F, Neukirchen D, Hindges R, Garvalov BK, Flynn KC, et al, Rac1 regulates neuronal polarization through the WAVE complex. J Neurosci. 2010;30:6930-43.

24. Zweier M, Rauch A. The MEF2C-related and 5q14.3q15 Microdeletion Syndrome. Mol Syndromol. 2012;2:164-70.

25. Phelan K, McDermid HE. The 22q13.3 Deletion Syndrome (Phelan-McDermid Syndrome). Mol Syndromol. 2012;2:186-201.

26. Engels $\mathrm{H}$, Wohlleber E, Zink A, Hoyer J, Ludwig KU, Brockschmidt FF, et al. A novel microdeletion syndrome involving 5q14.3-q15: clinical and molecular cytogenetic characterization of three patients. Eur J Hum Genet. 2009;17:1592-9.

27. Kleffmann W, Zink AM, Lee JA, Senderek J, Mangold E, Moog U, et al. $5 q 31$ microdeletions: definition of a critical region and analysis of LRRTM2, a candidate gene for intellectual disability. Mol Syndromol. 2012;3:68-75.

28. Kircher M, Witten DM, Jain P, O'Roak BJ, Cooper GM, Shendure J. A general framework for estimating the relative pathogenicity of human genetic variants. Nat Genet. 2014;46:310-5.

\section{Submit your next manuscript to BioMed Central and take full advantage of:}

- Convenient online submission

- Thorough peer review

- No space constraints or color figure charges

- Immediate publication on acceptance

- Inclusion in PubMed, CAS, Scopus and Google Scholar

- Research which is freely available for redistribution 